\title{
Electron propagation in orientationally disordered fullerides
}

\author{
E.J. Mele and S.C. Erwin \\ Department of Physics and Laboratory for Research on the Structure of Matter \\ University of Pennsylvania, Philadelphia, Pennsylvania 19104
}

(January 24, 1994)

\begin{abstract}
We study the electronic spectrum for doped electronic states in the orientationally disordered $M_{3} \mathrm{C}_{60}$ fullerides. Momentum-resolved Green's functions are calculated within a cluster-Bethe-lattice model, and compared with results from calculations on periodically repeated supercells containing quenched orientational disorder. Despite the relatively strong scattering from orientational fluctuations, the electronic states near the Fermi energy are well described by propagating states characterized by an effective Bloch wave vector, and a mean free path $\ell \approx 20 \AA$. The effective Fermi surface is calculated in this model. This differs from that previously calculated for the orientationally ordered crystal, but is relatively well described within a disorder-averaged virtual-crystal Hamiltonian, which we derive.
\end{abstract}

Typeset using REVTEX 


\section{INTRODUCTION}

Intercalation compounds formed by introducing alkali metals into the interstitial volume of the face-centered-cubic phases of the fullerenes have attracted a great deal of attention over the last several years [1]. The insulating undoped parent state can be doped into a metallic state by this method, and for the compounds $M_{x} \mathrm{C}_{60}$ with $x=3$ and $M=(\mathrm{K}, \mathrm{Rb})$ the ground state in these phases is superconducting. Since the first structural studies on these compounds by X-ray diffraction [2], it has been realized that the orientational structure of these doped phases is quite different from that of the undoped $\mathrm{C}_{60}$ solid. Undoped $\mathrm{C}_{60}$ undergoes a first-order phase transition from an orientationally disordered high-temperature phase to a lower-symmetry orientationally ordered four-sublattice structure at $\mathrm{T} \approx 250 \mathrm{~K}$ [3, 4 . In the $x=3$ doped phase, the intensities of the low-temperature diffraction peaks are well described by an orientationally disordered model. In this phase, each $\mathrm{C}_{60}$ molecule adopts a high-symmetry orientation with its two-fold axes aligned parallel to the [001] crystal directions. There are two inequivalent ways of achieving this orientation, which are related by a $\pi / 2$ rotation about any [001] axis. The intensities of X-ray reflections are well described by a model in which this choice of setting varies essentially randomly from site to site in the solid.

Orientational disorder has been cited as either a dominant or contributing factor to

many of the observed electronic properties of the normal and superconducting states of these doped systems [1]. The conduction electron states in these compounds are derived from an orbitally three-fold-degenerate state of the isolated molecule, with $t_{1 u}$ symmetry. The vector character of this state suggests that the intermolecular hopping amplitudes can be sensitive to the relative molecular orientations of neighboring $\mathrm{C}_{60}$ molecules. In fact, the hopping amplitudes between neighboring molecules are extremely sensitive to the molecular orientation, owing to the fact that they are dominated by hopping of electrons between the first several closest sites along a given intermolecular bond. This fact has been emphasized by Gelfand and Lu [5], and Satpathy et al. [6], who noted that because of this effect, the 
orientational disorder produces off-diagonal disorder in the one-electron Hamiltonian on an energy scale which corresponds approximately to the Fermi energy for the doped carriers in the $t_{1 u}$ conduction band. Some of the effects on this disorder on the transport properties and the frequency-dependent conductivity have since been explored [7,8].

Until quite recently, theoretical work on the electronic properties of the orientationally disordered phases has been limited to numerical simulations on supercells containing quenched orientational disorder. We recently reported a different approach to this problem, in which we developed an "effective medium" to describe the strong scattering effects in the orientationally disordered phases [8]. The theory is based on a cluster-Bethe-lattice approach in which the disordered medium is modeled by a three-band Bethe lattice, into which we embed a molecular cluster whose scattering properties are then studied. The scattering of the conduction electrons by the static orientational fluctuations in such a cluster can be accurately studied by this method with only modest computational effort. In Ref. [8], we demonstrated that this model successfully accounts for the structure of the one-electron spectrum, and for the low-frequency electronic excitations obtained from numerical work on large simulation cells.

Our calculations using this method demonstrate, somewhat surprisingly, that the effective elastic mean free path in the quenched disordered phases must extend though several coordination shells on the fcc lattice. It had been previously supposed that the strong fluctuations in the one-particle Hamiltonian would leave the merohedrally disordered solid in an extremely strong scattering regime for which the effective mean free path could not be meaningfully extended beyond a nearest-neighbor distance. This conclusion seems to have been supported to some extent by experimental measurements of the extrapolated residual resistivity, which have led to an estimate of the mean free path $\ell \approx 3 \AA$. By contrast, in Ref. [8] we found that the structure of the $t_{1 u}$ conduction band is accurately described only after the effects of phase-coherent propagation of electrons in closed rings of bonds through at least three coordination shells are retained in the calculations, so that one may estimate $\ell \approx 20 \AA$. This distance is sufficiently large that a k-space analysis of the conduction electron 
states is both possible and useful, as is the case for many other disordered alloys [9]. In this paper, we expand on this observation by developing this model in k-space and applying it to study the electron propagation in the orientationally disordered structure. As a point of comparison we will develop the analogous description for both a reference orientationally ordered doped phase, and for a model of a short-period ordered (but orientationally modulated) model. Our central result is that the conduction states for the quenched disordered phases do indeed show substantial, and quite possibly measurable, dispersion in k-space; this structure is very different from that found for the other ordered orientational alloys. We do find that the dispersion in the fully disordered phase can be successfully modeled by a "disorder-broadened" spectrum obtained from a particular ordered reference virtual crystal which we will describe. Finally, we point out that the dispersion in the single-particle spectral function might be directly accessible by an experimental probe such as angle-resolved photoemission. Such an experiment would be useful since it would provide both a spectroscopic probe of the orientational structure of the doped phases, and a test of the validity of the underlying one-electron picture adopted for these phases.

\section{EFFECTIVE MEDIUM THEORY}

In this section we provide a brief review of the Bethe-lattice construction which was originally reported in Ref. [8].

As noted in the Introduction, the conduction states in the alkali fullerides are derived from the orbitally three-fold-degenerate $t_{1 u}$ state of the isolated molecules. The broadening of this level into a conduction band can be described by introducing a nearest-neighbor $3 \times 3$ matrix hopping amplitude, $T_{\mu \nu}(\tau)$, describing the hopping amplitudes between states of orbital polarizations $\mu$ and $\nu$ across the bond $\tau$. The matrix elements in $T$ depend on the orientation of the bond $\tau$ and on the orbital polarizations. In their original work on this problem, Gelfand and Lu (GL) noticed that the signs and sizes of the various elements in $T$ depend on the relative molecular orientations on the terminal sites [5]. Their results for the 
matrix hopping amplitudes are reproduced in Table I.

The calculations of GL are carried out using a particular convention for labeling the orbital polarizations on each site, in which the coordinate axes are "fixed" in the frame of the molecule, and therefore spatially varying in the crystal frame (for a discussion of this point see Ref. [10]). It is useful to reformulate this result in a way which separates the effects of rotation of the coordinate system from the effect of variation of the underlying hopping amplitudes. A simple method is to construct a matrix amplitude $H_{\text {bond }}$ which describes the effects of forward and backward propagation across the bond $\tau$ in the manner

$$
H_{\text {bond }}(\tau)=\left(\begin{array}{cc}
0 & T_{M, N} \\
T_{M, N}^{T} & 0
\end{array}\right)
$$

Here $M$ and $N$ can take the values " $A$ " and " $B$ " denoting the orientation of the two sites connected by the hop. Squaring $H_{\text {bond }}$ in Eq. (1) decouples the two sites, demonstrating that the eigenvalues of Eq. (1) must be ordered in pairs $\pm\left|t_{n}\right|$, for any choice of the underlying matrix $T$. Each six-component eigenvector, $V$, of $H_{\text {bond }}$ can be then built up from two normalized three-component vectors $\left[u_{n}\right](n=i, j)$ in the manner $V=\left(u_{i}, u_{j}\right)$. For an eigenvector of the bond-hopping operator in the form $\left(u_{i}, u_{j}\right)$ with eigenvalue $e_{n}$, the vector $V^{\prime}=\left(u_{i},-u_{j}\right)$ is an eigenvector with eigenvalue $-e_{n}$. Thus the action of a general hopping matrix $T$ can always be reduced to the elementary $3 \times 3$ matrix operation $T=U_{j}^{T} h U_{i}$, where $h$ is a diagonal matrix containing (by convention) only the positive eigenvalues of $H_{b o n d}$ in Eq. (1), and $U_{i}$ is a matrix whose rows are the three components of the associated $V^{\prime}$ 's projected onto the $i$ th site. Here, the action of the internal hopping operator is positive definite, and all the sign and polarization conventions are contained in the $U$ rotation matrices.

The nonzero elements in the residual diagonal matrix $h$ can still depend on the relative molecular orientations. However, by a direct calculation of these, one finds that these nonzero diagonal matrix elements depend only weakly on the molecular orientation. As an example, we list in Table II the positive eigenvalues of $H_{\text {bond }}$ constructed for a hop between a bond between two molecules of like orientation (denoted $A A$ ), and two molecules of unlike 
orientation (denoted $A B$ ). For the most important, that is the largest of these, the fractional variation is only of order $10 \%$, indicating that the dominant effects of orientational disorder are introduced through the $U$ matrices and not through the bare amplitudes in $h$. The very slight variation of these eigenvalues in the two configurations occurs because in both the $A A$ or $A B$ orientations, a single bond is eclipsed along the nearest-neighbor direction. This single bond is labeled the " $5-6$ " bond since it borders a fivefold and sixfold ring on the surface of the fullerene. In the $A A$ orientation the fivefold and sixfold rings on neighboring molecules are eclipsed, while in the $A B$ orientation the fivefold ring eclipses a sixfold ring. In the construction of the effective medium model, we will therefore ignore the variation of the eigenvalues $h$ between the two structures and focus entirely on the very important orientation dependence of the rotation matrices $U$.

The effect of the rotations $U$ is to induce a reorientation of the orbital polarization of a given electron as it hops from molecule to molecule in the structure. If one regards the electron as a particle which carries with it an $\ell=1$ internal orbital degree of freedom, this Hamiltonian then describes a type of spin-orbit interaction coupling the orbital motion on the lattice to the internal orbital polarization of the particle. However, we emphasize that in this problem we are in a very strong scattering limit, where the reorientation of the orbital polarization is in no sense a "weak" precession of its internal moment as the particle propagates from site to site on the lattice.

The total density of states can be obtained from a trace of the one-particle Green's function $G_{+}(E)=(E+i \delta-H)^{-1}$, where the trace requires a sum over sites and over orbital polarizations on a given site. If one develops an expansion of the diagonal elements of $G$ in powers of $(1 / E) H$ :

$$
\begin{aligned}
G(E)= & \left(\frac{1}{E}\right)+\left(\frac{1}{E}\right)^{2} H+\left(\frac{1}{E}\right)^{3} H H \\
& +\left(\frac{1}{E}\right)^{4} H H H+\text { etc. }
\end{aligned}
$$

each term appearing in $\operatorname{tr} G$ can be interpreted as a specific closed electron trajectory on the lattice. For propagation in a disordered structure in which the $U$ 's are varying essentially 
randomly from bond to bond in the structure, one expects that large closed loops will not contribute spectral weight in the one-particle Green's function, since any reference orbital polarization will be scattered symmetrically into all possible orientations after its propagation on a closed loop. This cancellation rule fails for an orientationally ordered crystal since the large $N$-fold loops remain highly correlated for large $N$. Even in the orientationally disordered system, the special class of Brinkman-Rice [11] retraceable paths, which propagate randomly from some reference site and exactly retrace their steps back to the origin, always makes a positive-definite contribution to the trace for large $N$, and ultimately determines the long-distance behavior of the Green's function.

In view of this we are motivated to study the properties of an effective medium in which only the retracing paths are retained in the Green's function. For our problem, this network has the topology of a tree with one "ingoing" and eleven "outgoing" bonds at every node. The hopping amplitude along any single nearest neighbor bond on this network is given by the $3 \times 3 T$ matrices discussed above, which explicitly depend on the orientation of the bond. In the approximation that we neglect the weak dependence of the matrix $h$ on molecular orientation (see Table II), the intermolecular hopping amplitudes between like ( $A A$ or $B B)$ and unlike $(A B$ or $B A)$ orientations may be locally transformed into each other by suitable local rotations of the orbital bases. Of course, on a physical lattice this freedom will be eliminated by the closure of rings of bonds. On the tree, which contains no closed rings, we will consider the dynamics on a reference structure in which each site is arbitrarily assigned the " $A$ " orientation. Thus every node of the tree is equivalent, and one can then exploit this symmetry to construct the Green's function for the medium.

The construction of the Green's function on the tree is straightforward. The solution can be compactly expressed in terms of a complex matrix effective field $\Phi(E, \tau)$ which depends on energy $E$ and the bond orientation $\tau$ such that:

$$
G_{j, k}(E)=\Phi\left(\tau_{i j} ; E\right) G_{i, k}(E),
$$

where the subscripts denote lattice sites, and site $j$ is a nearest neighbor to site $i$. The 
Dyson equation for $E G(E)=I+H G$ provides a self-consistency condition for the field $\Phi$ :

$$
E \Phi(\tau)=H(\tau)+\sum_{\tau \neq \tau^{\prime}} H\left(\tau^{\prime}\right) \Phi\left(\tau^{\prime}\right) \Phi(\tau),
$$

from which $\Phi$ can be determined. In terms of the fields $\Phi$, the diagonal block of the oneparticle Green function at any site in the infinite tree is:

$$
G_{0,0}=\left[E-\sum_{\tau} H(\tau) \Phi(\tau, E)\right]^{-1} .
$$

The medium we construct in this manner will ultimately be used to provide a boundary condition for studying electron motion within (relatively small) orientationally disordered clusters extracted from the full orientationally disordered fcc lattice. Before turning to that application however, it is useful to examine the spectrum of the isolated tree, for which the density of states is shown in Fig. 目(a). (Here and elsewhere in the paper, all energies are expressed in units of $t$, which sets the energy scale for the matrix elements listed in Table I. Estimates of $t$ range between $10 \mathrm{meV}$ and $14 \mathrm{meV}$ for the doped systems.) We observe that this spectrum for the medium is an even function of energy, because all the retracing paths appearing in $G$ require exactly an even number of steps. The overall bandwidth obtained in this calculation is approximately $36 t$, which is very nearly the bandwidth obtained from direct numerical calculations on orientationally disordered supercells. This confirms the original conjecture that it is the retraceable paths which control the analytic structure in the one-particle Green's function for the disordered system. Finally, the spectrum is smooth and relatively structureless, reflecting the absence of closed coordination rings on the network. In fact, although the overall bandwidth agrees relatively well with that obtained from studies on disordered cells, the spectrum obtained for the tree is noticeably more symmetric and less structured than the spectra obtained from the numerical simulations on disordered lattices [8]. The structure obtained in the densities of state for the disordered lattices can be traced to the effects of the closure of the rings of bonds on the fcc network; these effects will be revealed more clearly by the momentum-space analysis given below.

We should remark that while the full matrix self-energy $\Sigma(E)=\sum_{i} H_{i} \Phi\left(\tau_{i}\right)$ is a complex diagonal $3 \times 3$ matrix (in orbital space) and therefore does not mix orbital polarizations, each 
of the individual contributions $\sigma_{i}=H_{i} \Phi\left(\tau_{i}\right)$ does have important nonvanishing off-diagonal components. These terms describe contributions to the on-site self-energy from indirect processes in which a particle, starting from a reference site, propagates into the tree and undergoes a reorientation of its orbital polarization before its return. The off-diagonal terms thus describe an orbital dephasing of the electron by its coupling to the external medium.

We now consider the effect of embedding a finite molecular cluster embedded in the disordered medium treated in this tree approximation. The full Hamiltonian is partitioned:

$$
H=H_{c}+H_{m}+H_{c m}
$$

where $H_{c}$ contains terms connecting two sites inside the cluster, $H_{m}$ refers to bonds connecting neighboring sites in the effective medium, and the $H_{c m}$ represents the connecting bonds at the boundaries of the cluster. The "internal" Hamiltonian $H_{c}$ is thus treated exactly in this formulation, and in particular the closure of coordination rings is completely accounted for within the molecular cluster in $H_{c}$. The Green's function for the composite medium can be projected onto this cluster by the matrix inversion:

$$
G(E)=\left(E-H_{c}-\sum_{i} \psi_{i}^{\dagger}\left(\sum_{j(i)} \sigma_{j}\right) \psi_{i}\right)^{-1},
$$

where the sum is over the boundary sites $i$ connected through the bonds $j$ to the external medium. Thus the effects of indirect propagation in the medium appear through the selfenergy contributions $\sigma_{j}$.

\section{DENSITIES OF STATES}

In this Section, we provide an analysis of the Green's function of Eq. (7) in momentum space. Before turning to those results, it is useful to examine the k-integrated total densities of states obtained for these systems.

In Fig. 1(b) we show the densities of states projected onto a single tetrahedral prism extracted from the fcc structure (the prism consists of four sites that are mutually nearest neighbors), embedded in the effective medium described above. Results are shown for 
the three inequivalent molecular configurations $A_{4}, A_{3} B$ and $A_{2} B_{2}$ within this tetrahedral cluster. One finds that the closure of the threefold rings of bonds perturbs the spectral structure obtained for the isolated tree. For the two configurations which contain bonds between molecules with inequivalent orientations, the effect is to provide a weak enhancement of the spectral weight at negative energy in the conduction band. However, it is also clear from the broad and relatively structureless character of the spectra that the electronic states in these clusters are dominated by the orbital dephasing introduced through coupling to the effective medium.

Similar calculations, in which the size and symmetry of the embedded cluster are varied, demonstrate that due to the large coordination number on the fcc lattice, the dephasing of the orbital polarization introduced by the effective medium is a very strong effect in this problem. Consequently, one must develop this model on relatively large embedded clusters before the data converge suitably to those obtained from numerical simulations on fully orientationally disordered lattices. As an example we reproduce, in Fig. Đ(c), data previously presented in Ref. [8] for embedded 19- and 43-site molecular clusters. These models just close the (002) and (112) coordination shells on the fcc lattice, respectively. Here we find that after closing the third coordination shell the spectra do provide an accurate representation of the spectra obtained from quenched orientationally disordered lattices (numerical results obtained from an average over an ensemble of 27-site lattices are shown for comparison). From this comparison one concludes that the single-particle Green's function is sensitive to phase-coherent propagation of the electrons over a range of order $\ell \approx 20 \AA$. Although relatively small by absolute standards, this length is surprisingly large given the observation that the scale of fluctuating term in the Hamiltonian for the $t_{1 u}$ electrons in the orientationally ordered phase is the one-electron bandwidth. However, the result can be rationalized by noting that these fluctuations appear only in the off-diagonal terms in the Hamiltonian, and can therefore be locally removed in any single bond by a gauge transformation to a suitable choice of orbital bases on two neighboring sites. To study the residual frustration to the electron motion we need to examine the propagation of the electrons on closed loops 
in the network; this can be described by a gauge-invariant correlation function.

\section{MOMENTUM SPACE FORMULATION}

In this section we reanalyze the results of Section III by formulating the theory in momentum space. This will be useful for understanding the propagation and the orbital structure of the conduction electron states, and for interpreting the various static-response functions which are mediated by the conduction electrons in the disordered phase.

Formally, our approach involves a simple rotation of the Green's function of Eq. (7):

$$
G(k, E)=\sum_{i, j} R_{i}^{\dagger}(k) G_{i, j}(E) R_{j}(k)
$$

with $R_{i}(k)=\exp \left(i k \cdot T_{i}\right)$ where $T_{i}$ is a lattice site locating the $i$ th molecular state. One observes that for a hypothesized model in the strong-disorder limit, for which the off-diagonal elements in the Green's function decay very quickly on the scale of a lattice spacing, only the diagonal elements of $G$ can contribute appreciable weight in the sum in Eq. (8), and therefore the projected $G$ is therefore approximately k-independent. The results of Section II of course indicate that the nonlocal terms in $G$ do contribute significantly even in our fully disordered model; the resulting dispersion of the spectral function $A(k, E)=(1 / \pi) \operatorname{Im} G(k, E)$ will be studied in this section. The calculated density $A(k, E)$ gives the probability that an electron at energy $E$ occupies a state indexed by crystal momentum $k$.

In Fig. 2 we plot the spectral function $A(k, E)$ calculated for wave vectors along the $\Gamma-X$ $\langle 001\rangle$ and $\Gamma-L\langle 111\rangle$ symmetry directions in reciprocal space, referred to the Brillouin zone of the reference fcc structure. These data are obtained carrying out the projection of Eq. (8) over a molecular cluster containing 79 sites. This closes the (310) shell around a reference site at the origin, with the boundary sites terminated by coupling to the Bethe lattice as

described earlier. These data are presented as a surface plot of $A(k, E)$ with $k$ plotted along one horizontal axis and the energy plotted along the orthogonal horizontal axis. In this plot, the states at negative energies are occupied when the conduction band is half filled. 
We find that the spectral function calculated in the model is dispersive, but broadened due to the scattering from the static orientational fluctuations. At the zone center, the spectral function is strongly peaked at an energy $E=-15.4 t$ relative to the Fermi energy. Along the $\Delta\langle 001\rangle$ direction this feature exhibits positive dispersion, so that at the zone boundary the spectral weight is evenly divided between positive- and negative-energy states. (Actually, a closer analysis reveals that this spectrum consists of two rather well resolved components, as shown in the inset of Fig. 2. For dispersion along the $\langle 001\rangle$ directions the $z$-polarized component of the $t_{1 u}$ band is essentially dispersionless, while the $x$ and $y$ components exhibit identical positive cosine-like dispersion. Along the $\Lambda\langle 111\rangle$ direction, these three orbital polarizations cannot be distinguished by their symmetry, and exhibit nearly identical positive dispersion away from the zone center. As we explain in detail below, all of these behaviors are predicted from the dispersion of an Hamiltonian for an orientationally averaged but ordered virtual crystal.)

For comparison, we have carried out the same calculation for an orientationally ordered reference cluster, results for which are shown in Fig. 3. The spectral function exhibits a dispersion which is by now quite familiar from the theory of $M_{3} \mathrm{C}_{60}$ in the fully orientationally ordered structure. The conduction band at $\Gamma$ shows a three-fold degenerate peak at a positive energy $E=8.2 t$ above the Fermi energy. This peak is split into an orbital doublet and singlet as one proceeds along the $\Lambda$ direction. Along the $\Delta$ direction, the $t_{1 u}$ multiplet is split into three separate states, two of which exhibit negative dispersion, and one of which disperses to higher positive energies. Since only the states at negative energies are occupied in the model, the band structure shown in Fig. 3 leads to a Fermi surface consisting of two interpenetrating sheets, corresponding to the two bands which cross $E=0$. The topology of this unusual Fermi surface is discussed in more detail in Refs. [12] and [13].

The widths of the spectral peaks in Fig. 3 are due to the finite size of the molecular cluster in which the calculations are carried out. Comparing the spectra in Figs. 2 and 3 we observe that the widths for the disordered phase are significantly larger than those obtained for the ordered model, and are therefore not "resolution limited", but are intrinsic to the 
orientational alloy.

Finally, we have carried out a similar calculation for the layered bidirectional structure, in which alternate layers are assigned uniformly to the $A$ and $B$ orientation, modulated along the [001] direction. The k-resolved spectral functions for this model are shown in Fig. 1 . (The axis conventions are the same as adopted in Figs. 2 and 3, but the Brillouin zone is now simple tetragonal.) Here we see more complex behavior, intermediate between that of the models presented in Figs. 22 and 3. The peaks in the spectral density are relatively sharp and strongly dispersive. The overall dispersion is positive, with a filled degenerate state at the zone center which then disperses above the Fermi energy as one approaches the Brillouinzone boundaries. The results obtained here agree in detail with the dispersion obtained by directly calculating the energies of the Bloch eigenfunctions propagating on a periodically repeated $A_{2} B_{2}$ crystal in the tetragonal structure. Note that the data presented in Fig. 4 use the conventions for the fcc primitive cell; that is they are plotted in an extended zone convention in which the six bands of the tetragonal crystal are unfolded into two groups of three which disperse along the $k_{z}$ direction.

\section{VIRTUAL CRYSTAL HAMILTONIAN}

The spectral function calculated for the orientationally disordered solid can be understood by studying the spectrum of a particular ordered reference crystal. The construction of this virtual crystal model as a reference Hamiltonian has been previously described in Ref. [10]. There the difference between the exact Hamiltonian in a particular orientational state and the virtual crystal was treated as a perturbation with which we were able to investigate the indirect orientation-dependent interactions between fullerenes mediated by the

response of the conduction electrons. Here we briefly review that construction and refer the reader to Ref. [10] for a more detailed discussion.

We observe that the orientation dependence of the nearest-neighbor matrix hopping amplitudes can be represented in the form 


$$
T_{M N}=t_{\mu, \nu}\left(\tau, \sigma_{M}, \sigma_{N}\right),
$$

where $\mu$ and $\nu$ run over the symmetry labels $x, y$, and $z$, and the $\sigma$ are Ising spin variables, where +1 denotes the $A$ orientation and -1 the $B$ orientation. The four possible hopping matrices between neighboring sites across bond $\tau$ can be neatly decomposed into an "average" contribution, two terms which are linear in the spin variables, and one term which is bilinear in the spin variables:

$$
t_{\mu, \nu}\left(\tau, \sigma_{M}, \sigma_{N}\right)=t_{\mu, \nu}^{(0)}+t_{\mu, \nu}^{(1)} \sigma_{M}+t_{\mu, \nu}^{(2)} \sigma_{N}+t_{\mu, \nu}^{(3)} \sigma_{M} \sigma_{N} .
$$

The virtual crystal is obtained by treating the $\sigma$ as uncorrelated random variables with $\langle\sigma\rangle=0$, so that the only nonvanishing term is $t^{0}$ in Eq. (10). This residual piece can be interpreted as resulting from an ensemble average over orientations, before relaxing the conduction electron states. Of course, since the electronic bandwidth is much larger than the frequency scale for orientational fluctuations, one should in principle reverse these two steps. However, we now show that the residual orientationally averaged ordered Hamiltonian successfully accounts for the symmetry and structure of the spectral functions shown in Fig. 2 for the orientationally disordered model.

For a hop along a (110) bond direction, symmetry considerations require that the virtual crystal $t^{(0)}$ takes the form

$$
t^{(0)}=\left(\begin{array}{ccc}
A & B & 0 \\
B & A & 0 \\
0 & 0 & C
\end{array}\right) .
$$

The values of $A, B$ and $C$ extracted from the GL Hamiltonian are given in Table III. Here we see that $A$ and $B$, which describe the hopping between $t_{1 u}$ orbitals which project along the bond direction $\tau$ (that is, the $x$ and $y$ polarizations) are quite small. This results from a near cancellation of hopping amplitudes which change sign as one flips the molecular orientations. The remaining constant, $C$, represents the hopping between $z$-polarized $t_{1 u}$ orbitals along the [001] direction, and dominates the intermolecular hopping amplitude. The analogous 
hopping matrices for motion along any of the bonds of the fcc structure can be obtained by a straightforward rotation of $t^{(0)}$ in Eq. (11).

The orientationally averaged matrix elements in Table III are interesting, since they suggest that it is useful to consider a simpler model in which we drop the small $x$ and $y$ hopping amplitudes, and consider the motion in the $x y$ plane, which is dominated by the propagation of a $z$-polarized conduction electron. (Similar arguments apply for propagation in the $y z$ and $x z$ planes.) Thus if we set the small amplitudes $A$ and $B$ listed in Table III to zero, we recover a reference Hamiltonian in which the orbital polarizations are completely decoupled in the model. The dispersion relations for these three bands are then given by the three independent spectra:

$$
\begin{array}{ll}
2\left(\cos \left(q_{y} a\right)+\cos \left(q_{z} a\right)\right), & x \text {-polarized } \\
2\left(\cos \left(q_{x} a\right)+\cos \left(q_{z} a\right)\right), & y \text {-polarized } \\
2\left(\cos \left(q_{x} a\right)+\cos \left(q_{y} a\right)\right), & z \text {-polarized }
\end{array}
$$

Eqs. (12) thus describe three interpenetrating dispersion relations for the $x-y$ - and $z$ polarized electrons propagating on three mutually orthogonal two-dimensional lattices. If we now reintroduce the smaller but nonvanishing elements $A$ and $B$, one finds a slight admixing of these branches, particularly along the $\langle 111\rangle$ directions, which induces a perturbation to this spectrum that is relatively well confined to $\mathrm{E}= \pm 2 t_{\|}$around the Fermi energy. The density of states for this virtual crystal is shown in Fig. 5, which clearly shows its underlying two-dimensional character.

The virtual-crystal approximation provides a surprisingly accurate description of the spectra calculated for the embedded clusters with quenched orientational disorder that were presented in Section III. The spectra shown there represent the spectral function for the disorder-averaged Green's function $\langle G\rangle$, while in the virtual crystal the ensemble average is applied first to the Hamiltonian, giving $G_{v c}=[E-\langle H\rangle]^{-1}$. Although these are clearly very different objects, the symmetry and structure of the one-particle states in the disordered model are represented reliably in the virtual-crystal approximation. 
The virtual crystal also correctly describes orbitally projected spectral densities of states obtained from the quenched average. For example, it is clear from Eq. (12) that the $z$ and $x y$ polarizations are decoupled for dispersion along the $\langle 001\rangle$ direction in k-space (and our observations above suggest that this should survive the reintroduction of the small amplitudes $A$ and $B$ ). In Fig. 6, the various orbital projections of the single-particle Green's function from the embedded cluster are displayed; they clearly exhibit exactly this behavior. The $z$-polarized component of the spectrum is nearly dispersionless, and is characterized by a prominent feature at the bottom of the $t_{1 u}$ conduction band. The $x y$-polarized components clearly exhibit a cosine-like dispersion along the $\Delta$ direction.

Finally, we turn to spectral densities at the fixed energy $E=E_{f}$, which is the alloy generalization of the crystalline Fermi surface. In Fig. 7 we show first the Fermi surface calculated for the orientationally ordered crystal (upper left), and the Fermi surface of the virtual-crystal model (upper right). For the virtual crystal, the Fermi surface can be described as intersecting two-dimensional "sheets". These are the extensions into three dimensions of the well-known inscribed square Fermi surfaces predicted by the square-lattice spectra of Eq. (12). The slight warping of these surfaces shown in the figure result from the weak nonzero elements $A$ and $B$ in the longitudinal block of $t^{(0)}$. By comparison we show, in the lower two panels of Fig. [7, the spectral function at fixed energy, $A\left(k, E_{f}\right)$, calculated in the effective-medium theory (lower left), and a direct numerical construction of $A\left(k, E_{f}\right)$ from Fourier analysis of the single-particle states at the Fermi energy, averaged over an ensemble of periodically repeated 27-site supercells with quenched orientational disorder (lower right). The structure of the virtual-crystal Fermi surface is clearly apparent in both of these plots. Moreover, the alloy Fermi-level momentum distribution can be described quite accurately by a disorder-broadened virtual crystal with an effective correlation length $\xi \approx 20 \AA$. This length is completely consistent with our analysis of the correlation length on the disordered fcc network given in Section III.

The accuracy of the virtual crystal theory can be understood by noticing that the resulting Fermi surface encloses the correct volume in phase space and exhibits the same $\left(O_{h}\right)$ 
symmetry as the full disorder-averaged Green's function. However, we should point out that while the virtual crystal correctly predicts the symmetry of the full spectral function, it does a poor job of quantitatively predicting the bandwidth. In fact, the bandwidth of the conduction band for the quenched disordered state is nearly a factor of two larger than predicted in the virtual crystal. These are compared in Fig. 5, as the solid (virtual crystal) and dashed (disordered crystal) spectra. Thus we observe that the fluctuating terms in the Hamiltonian do play a crucial role in fixing the scale of the intermolecular hopping amplitudes, but that the symmetries of the resulting spectrum are quite faithfully described by the annealed average in the simpler virtual-crystal approximation.

\section{DISCUSSION}

Orientational disorder in the fullerides introduces off-diagonal disorder in the effective electronic Hamiltonian for these alloys. Although the energy scale for this disorder is relatively large, we find that the scattering effects can be quite subtle. In particular, the orientation dependence of the interatomic hopping amplitudes can be essentially removed locally in any bond, by a suitable local rotation of the electronic basis states in the $t_{1 u}$ subspace. The residual effects of disorder then occur because the fluctuations in the Hamiltonian cannot be consistently removed globally in every bond by this procedure. It appears natural to formulate this problem as a gauge model on the fcc lattice, and indeed the moment expansion in Eq. (2) provides just such a formulation. Here we find that off-diagonal correlations in the one-particle Green's function are surprisingly robust, so that the states near the Fermi energy can be described as propagating states on an effective Fermi surface. Interestingly, this is different from what one obtains for the orientationally ordered crystalline phase.

At present, direct experimental data supporting the merohedral disorder hypothesis is obtained from X-ray scattering data. The idea is supported indirectly by the relatively large low-temperature resistivity, and by the observed breakdown of various selection rules for the 
excitation of the intramolecular vibrational modes. The dispersion of the spectral functions obtained for the disordered models studied in Section IV provide a direct microscopic probe of the scattering effects due to static orientational disorder. The overall dispersion of these bands is of order $0.5 \mathrm{eV}$, and might be directly resolvable by angle-resolved photoelectron spectroscopy. Indeed, the widths of these features in the spectral densities are similar to what one obtains in experiments on ordered simple metals. Since the sign of the dispersion is reversed in the ordered and disordered models, experimental observation of the dispersion of the single-particle spectral function can be used to test these models. Experimental observation of this dispersion might provide a useful test of the underlying one-electron picture which is commonly adopted to describe these phases. Of course this treatment ignores the effects of coupling to the vibrational degrees of freedom, as well as the effects of direct electron-electron interactions, which can also contribute to the observed single-particle spectra.

The conduction electrons in these systems also contribute to the effective potentials for the intramolecular vibrations in these doped solids. These are difficult to calculate accurately for the orientationally disordered systems. The most complete studies of these effects have made use of the supercell approximation in which the response functions are computed for a large, periodically repeated cell which contains orientational disorder on the fullerene sites. The effective-medium construction presented above can be straightforwardly applied to these studies, and provides an alternative method for investigating these effects.

Acknowledgements: This work was supported by the NSF under the grant DMR 91-20668 and by the Department of Energy under grant 91ER45118. 


\section{REFERENCES}

[1] A.F. Hebard, Physics Today, 11, 26 (1992)

[2] P.W. Stephens et. al, Nature 351, 632 (1991)

[3] P.A.Heiney, J.E. Fischer, A.R. McGhie, W.J. Romanow, A.M. Denenstein, J.P. McCauley, A.B. Smith and D.E. Cox, Physical Review Letters, 66, 2991 (1991)

[4] R. Sachidanandam and A.B. Harris, Physical Review Letters, 67, 1467 (1991)

[5] M.P. Gelfand and J.P. Lu, Physical Review Letters 68, 1050 (1992)

[6] S. Satpathy, V.P. Antropov, O.K. Anderson, O. Jepsen, O. Gunnarson and A.I. Lichtenstein, Physical Review B 46, 1773 (1992)

[7] M.P. Gelfand and J.P. Lu, Physical Review B 46, 4367 (1992)

[8] M.S. Deshpande, S.C. Erwin, S.Hong and E.J. Mele, Physical Review Letters 71 , 2619 (1993)

[9] B.L. Györffy and G.M. Stocks, in Electrons in Disordered Metals and at Metallic Surfaces, edited by P. Phariseau, B. L. Györffy, and L. Scheire (Plenum, New York, 1979), p. 89.

[10] T. Yildirim, S.Hong, A.B. Harris and E.J. Mele, Physical Review B (in press, October, 1993)

[11] W.F. Brinkman and T.M. Rice, Physical Review B2, 1324 (1970)

[12] S.C. Erwin and W.E. Pickett, Science 254, 842 (1991)

[13] E.J. Mele and S.C. Erwin, Physical Review B 47, 2948 (1993) 


\section{TABLES}

TABLE I. The intermolecular hopping matrices along a (110) bond direction for molecules in like $(A A)$ and unlike $(A B)$ orientations. The data are reproduced from reference [5]. The orbital polarizations are defined in the frame of the molecule, and differ by $\pi / 2$ rotations for the orientationally inequivalent case. The amplitudes are scaled by a factor $\mathrm{t} \approx 14 \mathrm{meV}$.

\begin{tabular}{lcccccc}
\hline \hline & & $A A$ & & & \\
& & & & & \\
& & & & & \\
& & $y$ & $z$ & & $y$ & \\
\hline$x$ & 0.83 & -1.98 & 0.00 & 1.75 & 2.08 & 0.00 \\
$y$ & -1.98 & 3.36 & 0.00 & -2.08 & -3.71 & 0.00 \\
$z$ & 0.00 & 0.00 & -1.91 & 0.00 & 0.00 & -2.67 \\
\hline \hline
\end{tabular}

TABLE II. The three positive eigenvalues of the bond hopping operator $H_{b o n d}$, for transitions between molecules with like $(A A)$ and unlike $(A B)$ settings. The eigenvalues of $H_{b o n d}$ are ordered in pairs $\pm|h|$.

\begin{tabular}{lr}
\hline \hline$A A$ & $A B$ \\
\hline 4.445 & 5.029 \\
1.910 & 2.670 \\
0.255 & 0.431 \\
\hline \hline
\end{tabular}

TABLE III. The three matrix elements which appear in the orientationally averaged virtual crystal defined by Eq. (11).

\begin{tabular}{lcc}
\hline \hline$A$ & $B$ & $C$ \\
\hline 0.01 & 0.38 & -2.29 \\
\hline \hline
\end{tabular}




\section{FIGURES}

FIG. 1. (a) The density of states calculated for the isolated fcc fulleride tree. Here and elsewhere in the paper the energy axis is scaled in units of $t$ which we estimate as $t \approx 14 \mathrm{meV}$. (b) The density of states traced over a tetrahedral cluster embedded in the tree. Three different orientational structures for the cluster are compared: $A_{4}, A_{3} B$, and $A_{2} B_{2}$. (c) The local density of states at the core of 19-site (dashed) and 43-site clusters (heavy solid) embedded in and coupled to the effective medium. The settings are chosen randomly in these clusters. Also shown (light solid) is the result of a quenched average over a series of 27-site orientationally disordered structures.

FIG. 2. Spectral function $A(k, E)$ for an orientationally disordered 79-molecule cluster coupled to the effective medium. The spectra are plotted with $k$ varying along the symmetry direction $L-\Gamma-X$ in the fcc zone. Inset: band structure for tight-binding Bloch states of the virtual-crystal Hamiltonian of Eq. (11).

FIG. 3. Spectral function $A(k, E)$ for an orientationally ordered 79-molecule cluster coupled to the effective medium. Plotting conventions are the same as adopted in Fig. 2. Inset: band structure for tight-binding Bloch states of the orientationally ordered fcc crystal.

FIG. 4. Spectral function $A(k, E)$ for an ordered bidirectional cluster coupled to the effective medium. The plotting conventions are the same as adopted in Figs. 2 and 3. Inset: band structure for tight-binding Bloch states of the bidirectional structure.

FIG. 5. The k-integrated density of states calculated for the orientationally averaged virtual crystal (solid) as described in the text. The dispersion is essentially two dimensional for each orbital polarization, with weak mixing between orbital polarizations. The density of states for the disordered crystal is reproduced from Fig. 11 as a dashed curve. 
FIG. 6. Orbitally projected spectral functions for the disordered system, dispersing along the $\langle 001\rangle$ directions in k-space. (Top) The $z$-polarized component is nearly dispersionless and peaked near the bottom of the conduction band. (Bottom) The $x$ - and $y$-polarizations show identical cosine-like dispersion, as found in the virtual-crystal approximation.

FIG. 7. Comparison of the Fermi-level momentum distributions $A\left(k, E_{f}\right)$ in the orientationally ordered state (upper left); the virtual-crystal Hamiltonian (upper right); the disordered state as described by the cluster-Bethe lattice model (lower left); and numerical simulations on disordered supercells (lower right). Note that the distributions for the disordered models are well described by a disorder-broadened version of the virtual crystal. 